\title{
A PERMANÊNCIA NO ENSINO SUPERIOR: UM ESTUDO SOBRE A REALIDADE DOS UNIVERSITÁRIOS DA UFT DESDE A PERSPECTIVA DA SUBJETIVIDADE
}

Permanence in higher education: a study on the reality of UFT University students from the perspective of Subjectivity

Permanencia en la educación superior: un estudio sobre la realidad de los estudiantes universitarios de la UFT desde la perspectiva de la subjetividad

\section{Katerine Silva Soares de Sousa ${ }^{* 1}$, José Fernando Patiño Torres ${ }^{2}$}

${ }^{1}$ Bolsista CNPq do Grupo de Pesquisa Subjectivus, Curso de Psicologia, Universidade Federal do Tocantins, Miracema do Tocantins, Brasil. Graduanda em Psicologia - UFT

${ }^{1}$ Grupo de Pesquisa Subjectivus, Curso de Psicologia, Universidade Federal do Tocantins, Miracema do Tocantins, Brasil. Psicólogo, mestre em psicologia cultural pela Universidade Del Valle (Colômbia) e doutor em educação pela Universidade de Brasília (Brasil). Professor do Programa de Graduação em Psicologia e Professor Visitante do Programa de Pós-Graduação em Comunicação e Sociedade da Universidade Federal do Tocantins (Brasil). Professor Visitante do Mestrado em Programa de Psicologia, Universidade de San Buenaventura (Colômbia). Professor Visitante do Curso de Especialização em Terapia Familiar e de Casais, Pontifícia Universidade Católica de Goiás (Brasil).

Artigo recebido em 10/04/2020 aprovado em 27/05/2020 publicado em 30/10/2020

\section{RESUMO}

O presente trabalho visa compreender os aspectos que envolvem a permanência no ensino superior com enfoque nas experiências subjetivas, assumindo uma perspectiva crítica sobre como os estudantes universitários têm vivenciado o processo de formação. Com esse propósito, foi realizado uma articulação teórica e em seguida, partiu-se para o levantamento de dados bibliográficos a partir da Teoria da Subjetividade de González Rey sob uma perspectiva sócio histórica. Em termos metodológicos, realizou-se uma pesquisa qualitativa que levou em conta três critérios epistemológicos fundamentais: o valor da singularidade dos participantes; a dialogicidade no trabalho de campo; e as construções interpretativas de natureza crítica e desnaturalizadora da realidade estudada. Para isso, foram convidados discentes universitários de vários cursos, os quais participaram de entrevistas individuais e grupos focais. $\mathrm{Na}$ discussão dos resultados, consideramos os aspectos subjetivos que levam o sujeito a permanecer ou não dentro deste contexto, incluindo relatos de vivência e as diferentes formas de subjetivação, aspectos estes, fundamentais para promover reflexões e estratégias que reconheçam o sujeito em sua integralidade em todos os aspectos que se relacionam com as diferentes dos processos humanos, elencando alguns pontos principais, tais como o distanciamento da família, a constituição dos laços sociais e a paixão pelo conhecimento.

Palavras chaves: Permanência em universitários; Processo de Formação; Subjetividade.

\section{RESUMEN}

El presente trabajo busca comprender los aspectos implicados en la permanencia en la enseñanza superior con enfoque en las experiencias subjetivas, asumiendo una perspectiva crítica sobre cómo los estudiantes universitarios han vivido el proceso de formación. Con ese propósito, fue realizada una articulación teórica y levantamiento de datos bibliográficos a partir de la Teoría de la Subjetividad de González Rey, sobre una 
perspectiva socio- histórica. En términos metodológicos, se realizó una investigación cualitativa que tuvo en cuenta tres criterios epistemológicos fundamentales: el valor de la singularidad de los participantes, la dialogicidad en el trabajo de campo y las construcciones interpretativas de naturaleza crítica $y$ desnaturalizadora de la realidad estudiada. Para esto, estudiantes universitarios de varios cursos fueron invitados, quienes participaron de entrevistas individuales y grupos focales. En la discusión de resultados, se consideraron los aspectos subjetivos que llevan al sujeto a permanecer o no dentro de ese contexto, incluyendo relatos de experiencia y las diferentes formas de subjetivación, aspectos fundamentales para promover reflexiones y estrategias que reconocen al sujeto en su integralidad en todos los aspectos que se relacionan con los diferentes procesos humanos, mencionando algunos puntos principales, tales como el distanciamiento de la familia, la constitución de lazos sociales y la pasión por el conocimiento.

Palabras clave: permanencia en universitarios, proceso de formación, subjetividad.

\section{ABSTRACT}

The present work seeks to understand the aspects involved in the permanence in higher education with a focus on subjective experiences, by assuming a critical perspective on how university students have lived through their educational process. For this purpose, from González Rey's Theory of Subjectivity, a theoretical articulation and collection of biographical data was carried out, by using a socio-historical perspective. In methodological terms, a qualitative research was carried out and took into account three fundamental epistemological criteria: the value of the singularity of the participants, the dialogicity in the field work and the interpretative-critical constructions of the reality studied. For this, university professors were invited, who participated in individual interviews and focus groups. In the discussion of results, the subjective aspects that lead the subject to remain or not within that context were considered, including stories of their experience and the different forms of subjectivation, fundamental aspects to promote reflections and strategies that recognize the subject in its integrality in all the aspects that are related to the different human processes, such as the distancing of the family, the constitution of social ties and the passion for knowledge.

Key words: permanency in higher education, educational process, subjectivity.

\section{INTRODUÇÃO}

O objetivo da presente pesquisa é promover reflexões acerca da permanência no ensino superior. Segundo ARAÚJO (2009), a ênfase na categoria permanência passou a ser considerada a partir dos anos 2000, através intensificação da instauração de políticas públicas voltadas para a expansão do ensino superior. Outro importante marco, foi o desenvolvimento de políticas que mostraram a necessidade de se estudar características específicas, tais como a importância do acesso e a permanência no ambiente acadêmico.

$\mathrm{Na}$ literatura que trata sobre o acesso às instituições de ensino superior no Brasil, mais especificamente às instituições públicas, observa-se que a categoria permanência pouco é abordada, e quando feito, é dirigido através da evasão escolar. Assim, ZAGO (2006), aponta que não basta ter acesso ao ensino superior, mesmo que seja público, se faz necessário que estudos sejam desenvolvidos a fim de compreendermos as condições da permanência.

No âmbito que tratam das políticas públicas, podemos destacar as políticas inclusivas desenvolvidas pelo Governo Federal, através do Ministério da Educação que, por meio do Plano Nacional de Educação (PNE), visa a inclusão social pela educação de todo o território nacional e tem entre outras metas, manter a oferta de vagas a pelo menos $30 \%$ dos jovens brasileiros. (MEC) 
Assim, a expansão do ensino superior, tornou-se um desafio para o estudo da categoria permanência, logo esta ação engloba maior acesso às universidades, inclusão de pessoas com deficiência, política de cotas e assistência estudantil e as desigualdades do sistema de ensino. Neste panorama de dificuldade, ZAGO (2006), aponta que não podemos desconsiderar os problemas estruturais que produzem as desigualdades escolares, por isso, o estudo com universitários possibilita conhecer, entre outras questões, a dinâmica que permeia a vida cotidiana e a formação universitária, como também as estratégias e o custo pessoal daqueles que procuram permanecer no sistema de ensino apesar das condições adversas de escolarização.

Observamos na literatura que se muito se debate sobre os aspectos objetivos inerentes da vida do sujeito, ou seja, fatores e indicadores imediatos e externos à vivência universitária - processo de escolarização anterior, moradia, alimentação, transporte, metodologias docentes que abarquem as diferentes culturas (acessibilidade aos povos originários e quilombolas), porém defendemos a necessidade de estudo dos aspectos subjetivos, inerentes da experiência individual e simbólica do sujeito que o leva a permanecer ou não dentro do contexto universitário.

Posto que é necessário que se observe as diferentes dimensões do êxito e do fracasso e os efeitos cumulativos advindo da própria subsistência bem como, dos efeitos da escolarização (ZAGO, 2006), se faz importante compreender os aspectos envoltos na formação universitária, tendo em vista que este processo de formação é uma etapa que proporcionará diferentes experiências e oportunidades que favorecem tanto a formação profissional quanto para o desenvolvimento psicossocial.

Por isso, propomos uma discussão desenvolvida à luz da Teoria da Subjetividade desenvolvida por González Rey por se constituir como uma teoria que busca compreender a subjetividade reconhecendo o valor da história e da cultura, possibilitando a emergência dos valores singulares do indivíduo. Posto que, a Subjetividade é entendida como dinâmica e complexa, em permanente mobilidade e permite a produção contínua de novos sentidos. (ROSSATO e MARTINEZ, 2013)

GONZÁLEZ REY (1997) define Subjetividade como a constituição psíquica do sujeito individual que integra processos e estados do mesmo em cada um de seus momentos de ação social, É uma produção simbólico-emocional singular, ou seja, a capacidade de gerar sentidos em todas as situações vividas. Esta teoria propõe a indissociabilidade entre indivíduo e social, rompendo com a clássica fronteira que separaria a psicologia, como ciência que se ocupa de um indivíduo universal a-histórico, das ciências sociais, como disciplinas que se ocupam exclusivamente dos processos coletivos (sociais, culturais, históricos), desconsiderando a dimensão singular da produção humana.

A teoria da Subjetividade avança em relação aos pressupostos teóricos consagrados em diferentes abordagens na psicologia, os quais tomam suas categorias como traduções a priori daquilo que é pesquisado. Assim, os desafios 
epistemológicos que o uso das categorias em discussão acarreta, apontam para processos de pensamentos não lineares, caracterizados pela reflexão, questionamento, curiosidade, confrontação teórica-metodológica frente ao pesquisado. (SOUZA e TORRES, 2019)

Esta pesquisa foi desenvolvida na Universidade Federal do Tocantins - UFT, Câmpus de Miracema, com participantes dos quatro cursos oferecidos pela unidade, sendo eles, Psicologia, Pedagogia, Serviço Social e Educação Física. Participaram do projeto, 06 acadêmicos que envolveram-se em entrevistas individuais e grupos focais que corroboraram para o levantamento das informações, onde foi possível observar os motivos que os levaram a ingressar em um determinado curso e o conhecimento que tinham sobre o curso que escolheram, bem como o posicionamento da família frente a escolha, a rede de apoio consolidada ou não e os enfrentamentos subjetivos para se manterem no contexto acadêmico.

\section{A permanência desde a perspectiva da} subjetividade: considerações teóricas de base

O Referencial conceitual a partir do qual se fundamenta a presente pesquisa é a Teoria da Subjetividade de GONZÁLEZ REY (2002b, 2005b, 2007, 2012, 2016, 2019) desde uma aproximação cultural-histórica (2002b, 2005b, 2007, 2012, 2016, 2019). Pensar o tema da permanência, desde a subjetividade, implica necessariamente entender a problemática enquanto fenômeno multicausal, complexo e perpassado pelas particularidades da cultura, da história e, principalmente, da forma como a experiência universitária é vivida pelo corpo discente.

A subjetividade, como categoria, é definida da seguinte forma:

A subjetividade, como domínio ontológico, especifica um novo tipo de processo qualitativamente diferente de todos os processos envolvidos em sua gênese. Assim, a subjetividade é definida ontologicamente pela integração de emoções e processos simbólicos, formando novas unidades qualitativas: sentidos subjetivos. Tais sentidos subjetivos são "instantâneos" de flashes emocionais simbólicos que se desdobram em um movimento caótico, do qual as configurações subjetivas emergem como uma organização auto-reguladora e autogeradora de sentidos subjetivos (GONZÁLEZ REY, 2019a, p. 28).

A subjetividade traz a importância, dentro dos processos de formação envolvidos na vivência universitária, da emoção na sua articulação com os processos intelectuais. É por isso que compreendemos, de entrada, que a permanência não depende de processos meramente racionais, cognitivos ou instrumentais. Permanecer dentro da trajetória universitária, com todas as suas variantes, é um verdadeiro processo subjetivo no qual o foco é a vivência singular da pessoa.

A emoção, dentro da psicologia e das ciências humanas em geral, tem sido uma categoria frequentemente esquecida pelo racionalismo 
instrumentalista que tem governado o pensamento moderno ocidental (GONZÁLEZ REY, 2013; PATINO, 2013; PATINO \& GOULART, 2016). Pensar na unidade que compõe o simbólico e o emocional implica reconsiderar de forma extensa a própria forma como temos compreendido a vida universitária a partir de olhares massificadores, adulto-cêntricos e mecanicistas.

Desde a perspectiva da subjetividade, viver a universidade é um processo que involucra pelos menos estes aspectos: as expectativas da família do/a discente; a presença/ausência da família, dentro da trajetória universitária; a forma de escolha do curso e sua correspondente motivação; a qualidade da relação com os/as docentes; a qualidade da relação com os/as colegas; a sociabilidade dentro dos câmpus; os aspectos objetivos para estudar: recursos para manutenção, segurança, saúde e alimentação; e a qualidade da cultura urbana na qual está inserido o câmpus.

Trata-se a permanência, então, como um fenômeno complexo e sistêmico que deve ser compreendido e promovido, de forma positiva, tendo em conta a forma singular em que se configura em cada indivíduo, turma, curso e/ou câmpus (PATINO, 2019; PATINO E CAIRES, 2018). A subjetividade permite pensar, também, que a permanência não é definitivamente uma questão do indivíduo, e sim da qualidade das configurações subjetivas da cultura e da história das instituições. Isso significa que assumir a permanência, como processo subjetivo, necessariamente requer uma compreensão ontológica que assuma o individual e o social como unidade de desenvolvimento. A articulação do social e do individual permite entender tanto a unicidade dos processos universitários dos/as acadêmicos/as, como também criar, a partir dos casos singulares, modelos teóricos que possibilitem generalizações com valor heurístico (GONZÁLEZ REY \& MITJÁNS MARTÍNEZ; PATINO \& GOULART, 2020). Dessa forma, o conceito de permanência, como expressão de uma produção singular, entrelaça a unidade entre o objetivo e o subjetivo.

Por fim, a subjetividade como arcabouço teórico nos permite recuperar, nas tramas da permanência universitária, ao sujeito como forma de resistência política e cultural. Desde a nossa perspectiva, o sujeito não é equivalente a indivíduo ou pessoa, mas sim um posicionamento subjetivo cuja qualidade, em termos ontológicos, se caracteriza por gerar rupturas e alternativas perante sistemas sociais e/ou individuais dominantes (González Rey, 2016; 2017; Patino \& Goulart, 2020). Em palavras de CAIRES E PATINO,

O sujeito emerge no exercício de suas elaborações singulares de pensamento frente o vivido. É nesse exercício, subjetivamente dinamizado, que $o$ desenvolvimento das possibilidades de ruptura com o instituído ganha força. Aqui, sublinha-se o pensamento como uma produção subjetiva, e não como exclusivamente uma construção racional. Isto não significa que toda forma de pensamento seja uma produção subjetiva. Implica que aqueles pensamentos emergentes no/do processo de subjetivação de uma relação social da pessoa, são 
desdobramentos de sentidos subjetivos de origens múltiplas, os quais, por sua carga simbólico-emocional, singularizarão as tomadas de decisões da pessoa (2019, p. 40).

Sujeito e subjetividade, por isso, são categorias teóricas subversivas, na medida em que nos permitem compreender a realidade social universitária, não como imposição e determinação, mas sim como produção subjetiva na qual as pessoas, grupos e coletivos tem capacidade para gerar mundos de subjetivação possíveis promotores de singularização.

\section{Permanência no Sistema de Ensino Superior}

Seguindo os avanços da expansão do ensino, os dados do Censo da Educação Superior de 2017, mostram que entre os anos de 2016 e 2017, a rede pública cresceu 2,8\% (Federal: 4,6\%; Estadual: 3,0\%; Municipal: $-17,2 \%)$. Assim, rede federal consolida-se como a maior rede pública com participação de $64 \%$ e com mais de 1,3 milhão de alunos em 2017. Nos últimos 10 anos, a rede federal teve a maior taxa de crescimento anual entre todas as redes, 7,4\%. (BRASÍLIA, 2018)

No que tange à permanência, o censo demonstra que dos 329.563 ingressantes, nas Instituições Federais de Educação Superior, em 2017, 69.256 (21\%) fizeram o Enem mais uma vez em 2017, mesmo já estando frequentando uma Instituição Federal de Ensino Superior - IFES. A partir desses números, o governo federal contabiliza que essa é uma evidência de que esses alunos buscam mudar de curso e/ou de Instituição, fenômeno que potencializa a desistência do curso e a criação de vagas remanescentes (BRASÍLIA, 2018). Ou seja, este é um dado que divulga um dos pontos considerados pelo Ministério da Educação como um fator de análise para a permanência no ensino superior.

No relatório do mesmo Censo analisado, um dado importante é que em 2017, 31.881 (10\%) dos novos alunos das instituições federais se matricularam em uma unidade da federação diferente daquela de sua residência, o que significa que esse quantitativo de ingressantes saíram de suas residências para residirem em outra unidade da federação, por conta dos estudos. Para nós, este ponto também é importante para a análise dos fatores da permanência e de como os acadêmicos são afetados por essas mudanças.

Para os alunos que se inserem no sistema de

ensino superior, o Ministério da Educação disponibiliza o programa Bolsa Permanência que se desenrola como uma política de auxílio financeiro para estudantes, sobretudo para os acadêmicos em situação de vulnerabilidade socioeconômica, indígenas e quilombolas, com a finalidade de minimizar as desigualdades sociais, étnico-raciais e no intuito de contribuir para a permanência e a consequente formação dos beneficiados. (FNDE, 2013).

Os dados apresentados acima, apontam que a permanência, é abordada segundo Araújo (2009), como a manutenção do estudante ao longo do curso, com vistas à sua terminalidade no tempo devido para sua integralização, observamos que a ampliação e o fortalecimento da rede de ensino que vêm acontecendo desde os anos 2000, certamente é 
de fundamental importância. A oferta de políticas sociais é importante, pois são a consolidação dos direitos sociais garantidos pela Constituição Federal de 1988. Porém, diante da intenção de investigar os aspectos subjetivos diretamente ligados à permanência no ensino é que discorreremos a seguir abordando questões importantes para o estudo desta categoria.

\section{MATERIAIS E MÉTODOS}

Este estúdio se realizou a partir da Epistemologia Qualitativa de GONZÁLEZ REY (1997, 2002a, 2005a; GONZÁLEZ REY \& MITJÁNS MARTÍNEZ, 2016, 2017A, 2017B, 2019; GONZÁLEZ REY \& PATINO, 2017; PATINO \& GOULART, 2020), a qual se fundamenta a partir de três princípios nucleares:

1) a singularidade como fonte legítima de conhecimento científico;

2) o diálogo como forma de comunicação dentro do trabalho de campo;

3) a metodologia construtivo-interpretativa para a geração de saber na pesquisa.

Este marco epistemológico para o estudo da permanência universitária enquanto processo subjetivo, implicou ir para além dos dados e das expressões objetivistas sobre as quais as pesquisas na área se têm focado: variáveis sociodemográficas, comportamento universitário, desempenho acadêmico, preditores de sucesso universitário, entre outros. Focar na subjetividade, para o presente trabalho, significou entrar nas produções singulares e íntimas de discentes no seu enfrentamento aos desafios universitários, ao tempo que gerar modelos teóricos que subsidiem explicações sobre o que acontece em relação com a permanência na formação superior.

Para tal fim, inicia-se então uma revisão bibliográfica a fim de construção do referencial teórico. Em um segundo momento foi realizado o levantamento da construção da informação para se pensar sobre a temática, por meio de entrevistas iniciais e, posteriormente, grupos focais. Através das informações construídas, foram realizadas construções interpretativas para responder aos objetivos propostos desta pesquisa.

A pesquisa foi realizada no campus Universitário de Miracema do Tocantins, convidando no trabalho de campo a 06 participantes jovens universitários de 18 a 27 anos, que residiam em Miracema. Vale ressaltar que todos os participantes recebem auxílios para se manterem no curso e são oriundos de estados diferentes, sendo um da região sul do país, dois do centro-oeste e 4 distribuídos nos estados da região norte. Visando o sigilo total e a não exposição dos conteúdos trazidos pelos participantes, os nomes não serão divulgados.

Os participantes da pesquisa foram escolhidos a partir de alguns critérios estabelecidos:

- Estar matriculado em algum curso de ensino superior do campus de Miracema.

- Ter idade entre 18-27 anos.

- Participar voluntariamente das etapas desta pesquisa.

Nas aproximações com os participantes, a equipe de pesquisa se reunia para dialogar sobre os detalhes do projeto: seus objetivos, alcances, metodologia e tratamento ético das pessoas e informações. 
Posteriormente, depois dos participantes estarem cientes e de acordo com os parâmetros do estudo, realizávamos as entrevistas e grupos focais, segundo a disponibilidade deles. Por último, se realizava a transcrição dos diálogos de campo produzidos, se sistematizavam dentro das categorias emergentes no processo construtivo-interpretativo e depois se realizava a escrita dos relatórios e artigos.

\section{RESULTADOS E DISCUSSÃO}

Quando falamos em permanência no ensino superior, os dados estatísticos nos chamam a atenção, pois apenas mencionam o fenômeno como uma questão social. Além disso, tratam a problemática como um fenômeno de fatores mecânicos que determinam que pessoa permanece na trajetória universitária, e qual não. O problema da epistemologia dos fatores, tão comum nos estudos sobre formação universitária, é que assumem que a permanência é um epifenômeno dos fatores externos, a saber: origem socioeconômica, capital cultural, apoio familiar, qualidade pedagógica, entre outros. Neste caso, nos distanciamos dessas aproximações para pensar melhor a forma em que as dimensões que constituem a permanência estão configuradas de forma singular, tanto nos indivíduos, como nos coletivos universitários.

Diante disto, consideramos as diversas situações emergentes dos participantes a partir do entendimento de que cada indivíduo carrega consigo diferentes relações afetivas, responsabilidades e incertezas diante do futuro. Compreender estas características, nos permitiu refletir sobre os aspectos que envolvem a escolha do curso, a relação familiar frente a escolha, o sentimento de pertença e a rede de apoio como elementos fundamentais que permitam ao indivíduo permanecer na universidade. Esses aspectos, mais do que serem fatores causais, são elementos que compõem a configuração do processo subjetivo da permanência. Assim, por exemplo, para um/a discente o apoio familiar pode ser elemento negativo e pouco dinamizador, porém a rede de apoio desenvolvida na trajetória universitária é tão forte que consegue tributar, de forma favorável, a uma adequada permanência na formação.

Da mesma forma, avançar para além dos dados estatísticos, nos permitiu entrar em contato com situações que estão implicadas na escolha de permanecer. Nos grupos, observamos em uma das falas que o participante relata estar descontente com o curso no qual estava matriculado, pelo que resolve cancelar a matrícula para iniciar outro curso, sem conhecimento prévio das características da nova área de estudo e de trabalho.

"Eu cursava direito, mas não gostei. Eu odiava as aulas. E tranquei e vim para o curso de psicologia. Mas não imaginava o que era a psicologia" (estudante do curso de psicologia, 21 anos).

Não se sentir pertencido a uma área de estudo, nos permite observar que, para além da escolha, o sentimento de pertença também é um aspecto que leva os indivíduos a buscarem outras alternativas. Queremos destacar aqui uma questão de relevância: a maioria dos/as discentes universitários ingressam aos cursos, sem ter passado por experiências formativas (seja na escola, 
seja no início da universidade) que lhes permitissem três ações fundamentais para realizar uma escolha profissional:

1) Procurar informações, pessoas e experiências necessárias para conhecer a fundo os potenciais cursos;

2) Refletir sobre suas motivações em relação com as possíveis áreas de conhecimento e futura atuação profissional;

3) Confrontar motivação e capacidade, para saber se há ou não algumas garantias de sucesso para enfrentar um determinado curso. Tanto escola como universidade são omissas e indiferentes perante a situação dos/as jovens que, cada uno, se enfrentam a uma escolha tão importante para as suas vidas.

Outro ponto que queremos ressaltar é que, no momento em que um/a discente decide trocar de curso, o faz de novo com grande desconhecimento sobre a nova área, o que é um gerador potencial de mal-estar e possível sofrimento. Mesmo que sair de um curso sobre o qual não se tem motivação pode ser uma alternativa interessante para uma nova vivência subjetiva a partir da possibilidade da emergência da carreira acadêmica, de novo a pessoa não tem garantias de que está escolhendo com os recursos necessários para não repetir a situação que já passou pelo curso anterior.

A escolha do curso e da universidade implicam outros aspectos. A dinâmica e a história familiar também colaboram no processo de escolha. A partir da teoria da subjetividade, entendemos que a família assume um significativo lugar no desenvolvimento subjetivo. No fragmento a seguir, observamos que a família impõe uma barganha e mostra que os recursos financeiros são escassos, ou seja, para escolher convém analisar as possibilidades dentro de um único campo - o da instituição pública, e ainda demonstram sua expectativa neste processo.

“Minha mãe disse: 'você passa no ENEM, por que eu não tenho dinheiro para pagar. $\mathrm{E}$ depois de se formar, tem que puxar a seus irmãos mais novos" (estudante de serviço social).

A relação estabelecida com o primeiro grupo social, a família, possibilita ao indivíduo diferentes compreensões subjetivas dos diferentes processos da vida. Essa relação permite que os indivíduos possam se organizar com os elementos que envolvem compreensão, desenvolvimento e interação, ou seja, é na inter-relação com os grupos é que se faz possível reconhecer-se como pessoa ativa e criativa nas suas escolhas.

Para o caso do trecho anterior, podemos ver a família coloca uns parâmetros a partir dos quais o jovem pode organizar a suas possibilidades a futuro. Nesse sentido, não só tem que fazer uma excelente prova no ENEM, mas também tem que visualizar que há um objetivo que está para além da formatura: "puxar a seus irmãos mais novos". Isso nos permite corroborar, como foi colocado em estudo anterior (Patino, 2012), que a moratória social da juventude universitária não se vive de forma banal e despreocupada. Muito pelo contrário, os/as jovens brasileiros/as das universidades federais tem enormes exigências acadêmicas e familiares, o que muitas vezes acabam se constituindo como configurações subjetivas geradoras de sofrimento. Se levamos em consideração que mais do $80 \%$ da 
população estudantil da UFT tem algum grau de vulnerabilidade social, isso nos obriga a considerar seriamente que a formação universitária é um processo muito mais complexo que simplesmente uma educação bancária, conteudista e irreflexiva.

Nessas situações que experimentam os/as universitários/as, a criatividade se faz necessária. É preciso que haja uma espécie de validação que seja capaz legitimar o indivíduo e seu lugar nas escolhas que faz, o que não impede que esse processo seja desprovido de acompanhamento por parte dos atores das universidades. As instituições federais, em muitos dos casos, assumem um papel omisso e violento quando desconsideram oferecer acompanhamento a discentes para fortalecer a sua permanência no cenário universitário, sob uma premissa: "Aqui só sobreviverão os mais fortes". Esta expressão, escutada pela nossa equipe durante a pesquisa, nos mostra que boa parte da universidade está comprometida, não com uma formação significativa, digna e emancipadora, mas sim com um darwinismo social anacrónico que pretende eliminar aos mais vulneráveis.

As incertezas e sentimentos de não pertencimento, muitas vezes levam a discentes a abandonarem os cursos, sem conseguir falar com alguém que os possa orientar nesse momento crítico:

“Às vezes eu penso: será que é isso mesmo que eu quero? Aqui nunca temos um espaço para pensar. Temos que estudar e produzir, o tempo todo" (estudante de psicologia).

Este trecho nos mostra como a universidade entrou em um processo de produção que é característico das fábricas das primeiras etapas do capitalismo. Um processo em massa e para as massas, que desconsidera a singularidade do processo e as pessoas envolvidas nele, devido a que seus valores de existência estão relacionados com uma irreflexiva produtividade que não produz, finalmente, nada. $\mathrm{O}$ caso anterior nos ensina algo interessante: é uma estudante que está questionando sobre sua motivação e, ao mesmo tempo, criticando que não há tempo para pensar. Uma universidade que não pensa? É possível isso? Os casos alarmantes e crescentes de suicídios, a nível nacional, nos mostram o desdobramento de uma vida sobre a qual nada se pode pensar nem refletir.

González Rey (2019), discorre em sua teoria que o desenvolvimento da subjetividade ocorre em diferentes processos de subjetivação. A universidade está obrigada, desde a sua missão social, a favorecer, através de recursos humanos, processos, atividades e infraestrutura, desenvolvimentos subjetivos que possibilitem nos/as discentes novas experiências curriculares extracurriculares. Quando falamos de desenvolvimento subjetivo não se trata de apreender conhecimentos de forma mecanicista e desprovida de motivação e ética do sujeito. Melhor, se trata de construir uma formação que possibilite que cada pessoa possa emergir como sujeito da sua formação universitária, o que implica assumir uma posição autoral e motivada em relação com o que estuda.

Queremos finalizar comentando que, perante a nova pandemia do covid-19, a universidade está exortada a criar dispositivos que permitam o tratamento adequado dos desdobramentos subjetivos que vão acontecer. A 
universidade deve se concentrar em refletir sobre qual é seu papel dentro da sociedade. Este estudo tem sido uma oportunidade para confrontar o que os atores da universidade estamos fazendo, com o intuito de responder a seguinte pergunta: qual é a universidade que podemos imaginar para este novo mundo?

\section{CONCLUSÃO}

A revisão da literatura nos mostrou que há uma escassez de pesquisas que reconheçam os aspectos subjetivos dentro do processo universitário e da sua correspondente permanência. A hegemonia dos estudos está centrada em fatores de determinação, o que acaba empobrecendo o debate da permanência por ser considerada apenas um epifenômeno de preditores sem valor teórico nem empírico.

Para o estudo da categoria permanência, observamos que muitos posicionamentos devem ser considerados. O fundamental, para o presente trabalho, é reconhecer o caráter subjetivo que permanência tem no seu processo constitutivo. Nosso propósito não está em afirmar o melhor e mais eficiente no estudo da categoria, mas compor um arcabouço teórico que permita uma ampliação de diferentes reflexões que possibilitam distintas formas de operacionalização das informações, bem como de projetos de pesquisa e extensão que sejam favorecedores de desenvolvimento subjetivo em sua total integralidade.

Certamente, este processo nos permitiu desenvolver novos questionamentos sobre a permanência, pois compreendemos que as dificuldades enfrentadas pelos acadêmicos não se fundam apenas na construção social como uma realidade externa, mas que toda a vivência do sujeito, tais como o processo de escolarização, as redes de apoio, a relação familiar são importantes para que possamos de fato entender como o indivíduo se consolida na escolha de permanecer no ensino superior.

Por se tratar de um fenômeno complexo, expomos nesta pesquisa, alguns processos simbólicos-emocionais que impactam nas diferentes escolhas individuas e que acarretam diferentes processos subjetivos, a fim de problematizar o reconhecimento da integralização dos/as universitárias. Consideramos, por fim que esta pesquisa possa ser um condutor que possibilite a professores, acadêmicos e comunidade reconhecer as distintas nuances, bem como a formulação de propostas de apoio discente que leve em consideração a complexidade do ser, do estar e do permanecer no cenário universitário.

\section{AGRADECIMENTO}

"O presente trabalho foi realizado com o apoio do Conselho Nacional de Desenvolvimento Científico e Tecnológico - CNPq - Brasil"

Todos os autores declararam não haver qualquer potencial conflito de interesses referente a este artigo.

\section{REFERÊNCIAS}

ARAÚJO, C.B. ZANDAVALLI M. A permanência de Estudantes no curso de graduação no Brasil: Uma categoria em construção. Revista Temas em Educação. João Pessoa, v.22, n.2, p. 25-43, jul.dez. 2013. 
BRASIL. Ministério da Educação. Fundo Nacional de Desenvolvimento da Educação. Bolsa Permanência. Brasília. Disponível em: https://www.fnde.gov.br/index.php/programas/bols as-e-auxilios/eixos-de-atuacao/bolsa-permanencia. Acesso em 31 março 2020.

BRASIL. Ministério da Educação. Instituto Nacional de Estudos e Pesquisas Educacionais Anísio Teixeira. Censo da Educação Superior de 2017. Brasília, 2018.

GONZÁLEZ REY, F. Epistemología cualitativa y subjetividad. São Paulo: Educ, 1997

GONZÁLEZ REY, F. Pesquisa qualitativa em psicologia: caminhos e desafios. São Paulo: Thomson Learning. 2002a.

GONZÁLEZ REY, F. La subjetividad: su significación para la ciencia psicológica. In O. Furtado, F. González Rey (orgs.). Por uma epistemologia da subjetividade: um debate entre a teoria sócio histórica e a teoria das representações sociais. São Paulo: Casa do Psicólogo. 2002b.

GONZÁLEZ REY, F. Pesquisa qualitativa e subjetividade: Os processos de construção da informação. São Paulo: Thomson. 2005a.

GONZÁLEZ REY, F.). Fernando. Sujeito e subjetividade: uma aproximação históricocultural. Tradução: Raquel Souza Lobo Guzzo. São Paulo: Pioneira Thomson Learning. 2005b.

GONZÁLEZ REY, F. As categorias de sentido, sentido pessoal e sentido subjetivo: sua evolução e diferenciação na teoria histórico-cultural. Psicologia da Educação. São Paulo, 24, 1 sem. 2007.

GONZÁLEZ REY, F. Subjetividad social, sujeto y representaciones sociales. Revista Diversitas Perspectivas en Psicología, Vol. 4, No. 2, pp. 225243. Universidad Santo Tomás. 2008.

GONZÁLEZ REY, F. Epistemología y ontología: un debate necesario para la psicología hoy. In: Diversitas: Perspectiva Psicológica. Vol. 5, No. 3, 205-224. 2009.
GONZÁLEZ REY, F. Las categorías de sentido, sentido personal y sentido subjetivo en una perspectiva histórico-cultural: un camino hacia una nueva definición de subjetividad. Universitas Psychologica, Vol. 9, No. 1, pp. 241-253. Pontificia Universidad Javeriana. 2010.

GONZÁLEZ REY, F. Fernando. Advancing on the concept of sense: subjective sense and subjective configurations in human development. In $\mathrm{M}$. Heideggard, A. Edwards, M. Fleer (orgs). Motives in children's development: cultural-historical approaches. 1ed. Cambridge: Cambridge University Press, v.1, pp 45-62. 2012.

GONZÁLEZ REY, F. O que oculta o silêncio epistemológico da psicologia? Pesquisas e Práticas Psicossociais. 8(1), 20-34. 2013.

GONZÁLEZ REY, F. Advancing the topics of social reality, culture and subjectivity from a cultural-historical standpoint: moments, paths and contradictions. Journal of Theoretical and Philosophical Psychological. Vol.36, No. 3, 175189. 2016.

GONZÁLEZ REY, F. The topic of subjectivity in psychology: Contradictions, paths and new alternatives. Journal for the Theory of Social Behaviour, v. 47 , n. 7 , p. 502-521. DOI: org/10.1111/jtsb.12144. 2017.

GONZÁLEZ REY, F. Subjectivity as a new theoretical, epistemological, and methodological pathway within cultural-historical psychology. In F. González Rey, A. Mitjáns Martinez, D. Magalhães Goulart (Orgs). Subjectivity within culturalhistorical approach. Perspectives in culturalhistorical research. Singapore: Springer, 2019.

GONZÁLEZ REY, F. \& MITJÁNS MARTÍNEZ, A. Una epistemología para el estudio de la subjetividad: sus implicaciones metodológicas. Psicoperspectivas (Online): Individuo y Sociedad, 15(1), 5-16. 2016.

GONZÁLEZ REY, F. \& MITJÁNS MARTÍNEZ, A. Subjetividade: epistemologia, teoria e método. Campinas: Alínea. 2017a.

GONZÁLEZ REY, F.; MITJÁNS MARTÍNEZ, A. Epistemological and methodological challenges for

Revista Desafios -v. 7, Especial - PIBIC, 2020 
the study of subjectivity from a cultural-historical perspective. In M. Fleer; F. González Rey; N. Veresov (Orgs.), Perezhivanie, emotions and subjectivity: Advancing Vygotsky's legacy. New York: Springer, 264-296. 2017b.

GONZÁLEZ REY, F. \& MITJÁNS MARTÍNEZ, A. The constructive-interpretative methodological approach: orienting research and practice on the basis of subjectivity. In: F. González Rey, A. Mitjáns Martinez, A. \& D. Goulart. Theory of subjectivity: new perspectives within social and educational research. New York: Springer, 37-60. 2019.

GONZÁLEZ REY, F. \& PATINO, J.F. La epistemología cualitativa y el estudio de la subjetividad em una perspectiva cultural-histórica. Conversación con Fernando González Rey. Revista de Estudios Sociales, 60, 120-128. DOI: https://doi.org/10.7440/res60.2017.10. 2017.

PATIÑO, J.F. La juventud: una construcción social histórica de Occidente. En: Revista Guillermo de Ockham, Vol. 7, No. 2., Universidad de San Buenaventura - Cali. 2009.

PATIÑO, J.F. Jóvenes universitarios contemporáneos: contradicciones y desafíos. 1. ed. Cali: Editorial Bonaventuriana, 2012.

PATIÑO, J.F. Del medioevo a la modernidad iniciática: una perspectiva histórico-cultural. Fragmentos de Cultura (Goiânia), v. 23, p. 229244. 2013.
PATIÑO-TORRES, J.F. A relação orientador-aluno na formação doutoral: uma aproximação a partir da Teoria da Subjetividade. In: M. Rossato e V. Peres. Formação de educadores e psicólogos. Contribuições e desafios da subjetividade na perspectiva cultural-histórica. Curitiba: Appris. 2019.

PATIÑO-TORRES, J.F. \& Caires, E. Discussing subjectivity in undergraduate and graduate education. In: González Rey, F., Mitjáns Martinez, A., \& Goulart, D. Theory of subjectivity: new perspectives within social and educational research. New York: Springer. 2018.

Patiño, J.F. e Goulart, D. Qualitative Epistemology: A scientific platform for the study of subjectivity from a cultural-historical approach. International Research in Early Childhood Education, 7(1), 161-181. 2016.

ROSSATO. M. e MITJÁNS MARTÍNEZ, A Desenvolvimento da Subjetividade: análises de histórias de superação das dificuldades de aprendizagem. Revista Semestral da Associação Brasileira de Psicologia Escolar e Educacional, 17(2), 289-298, 2013.

CAIRES, E E PATIÑO, J. F. A teoria da Subjetividade e seus conceitos centrais. Obutchénie: R. de Didat. e Psic. Pedag. 3 (1), 3457, 2019.

ZAGO, N. Do acesso à permanência no ensino superior: percursos de estudantes universitários de camadas populares. Revista Brasileira de Educação, 11(32), pp. 226 - 237, 2006. 